\title{
EFEITOS DA SEQUÊNCIA DE BRANQUEAMENTO NAAÇÃO DE ALVEJANTES ÓTICOS EM CELULOSE KRAFT DE EUCALIPTO
}

\author{
Mauro Manfredi ${ }^{1 *}$, Jorge Luiz Colodette $^{1}$, Rubens Chaves de Oliveira ${ }^{1}$, Bianca Moreira Barbosa ${ }^{1}$ \\ *Autor para correspondência: mauro.manfredi@ufv.br
}

\begin{abstract}
RESUMO: Durante o processo de branqueamento a celulose é tratada com reagentes químicos que, ao se tornarem resíduos na polpa, podem interferir na ação dos alvejantes óticos. Diferentes sequências de branqueamento podem produzir polpas com o mesmo grau de alvura, mas com potenciais diferentes para incremento da brancura por meio da aplicação de alvejantes óticos. Neste trabalho, objetivou-se avaliar a influência da sequência de branqueamento da celulose na eficiência de alvejantes óticos dos tipos dissulfonados e tetrassulfonados. Foram utilizadas polpas kraft de eucalipto branqueadas por quatros sequências de branqueamento em três níveis de alvura. Para cada sequência de branqueamento foi gerado um modelo matemático predizendo a brancura final da polpa em função da alvura inicial e carga (quantidade de reagente) de alvejante aplicada. Foi constatado que a presença de resíduos organoclorados na celulose reduz a eficiência dos alvejantes. Sequências que utilizam menor carga de dióxido de cloro possibilitam maiores ganhos na brancura com a aplicação de alvejantes óticos. A substituição de um estágio final de branqueamento com dióxido de cloro por um estágio com peróxido de hidrogênio aumentou a eficiência dos alvejantes.
\end{abstract}

Palavras-chave: alvejante ótico, branqueamento, celulose de eucalipto.

\section{EFFECTS OF THE BLEACHING SEQUENCE ON THE OPTICAL BRIGHTENERS ACTION IN EUCALYPTUS KRAFT PULP}

\begin{abstract}
During the bleaching process the pulp is treated with chemical reagents that can be retained in the pulp and interfere in the action of the optical brighteners. Different bleaching sequences can produce pulps at the same brightness but with different potential to whiteness increase when treated with optical brighteners. The objective of this study was to evaluate the influence of the bleaching sequence on the efficiency of disulphonated and tetrasulphonated optical brighteners. Eucalyptus kraft pulp was bleached using four different bleaching sequences. For each pulp three brightness targets were aimeds. For each bleaching sequence mathematical model was generated for predicting the final pulp whiteness according to the initial brightness and the optical brightener charge applied. The presence of organochlorine residues in the pulp reduced the effectiveness of the optical brighteners. Therefore, bleaching sequences that use low chlorine dioxide charge favors for greater gains in whiteness with the application of optical brighteners. The replacement of the final chlorine dioxide bleaching stage with a hydrogen peroxide one in the sequence increased the efficiency of the optical brightening agents.
\end{abstract}

Keywords: Optical brighteners, bleaching, eucalyptus pulp.

\section{INTRODUÇÃO}

Alvura e brancura são propriedades da celulose que expressam o quanto branco a celulose se apresenta. A alvura é calculada baseada na refletância apenas de radiação nos comprimentos de onda próximos à $457 \mathrm{~nm}$ (faixa do azul), enquanto que a brancura considera a refletância em toda a região do visível, que vai de 400nm à 700nm (JORDAN, 1996).

A alvura é muito utilizada pelas indústrias de celulose. Por se tratar de um material amarelado, a celulose absorve radiação violeta e azul-violeta (LEONARDI, 1990). Ao remover os compostos que conferem a coloração amarelada, o processo de branqueamento aumenta a reflexão de radiação na faixa do azul, o que torna a análise de alvura eficiente para o controle do processo de branqueamento (DENCE; REEVE, 1996).

As indústrias de papel necessitam de um controle mais rigoroso das características óticas do seu produto. Portanto, utilizam a análise de brancura, que ao avaliar a reflectância em toda a região do visível, expressa um valor para o branco mais coerente com a percepção do olho humano.

Para atingir elevado grau de brancura, a celulose pode ser tratada com alvejante ótico. Segundo Pelissari et al. (2003), dependendo da composição da polpa, os alvejantes óticos são responsáveis por 25 a $35 \%$ da brancura final do papel. O branqueamento ótico é considerado como o

1 Universidade Federal de Viçosa - Viçosa, Minas Gerais, Brazil

Cerne, Lavras, v. 20, n. 2, p. 223-230, abr./jun. 2014 
método mais moderno para a compensação da tonalidade amarelada do substrato (FORSSKALH, 2000).

Em princípio, o alvejante ótico é um corante fluorescente que absorve parte da radiação ultravioleta incidente, aumenta o seu comprimento de onda pela fluorescência e a reflete na forma de luz azulada. Tal fenômeno aumenta a quantidade de luz visível emitida pelo papel. A luz fluorescente azulada compensa a tonalidade amarelada natural da celulose, resultando assim num nível de claridade mais alto do que a cor original (ALÉN, 2000).

Os alvejantes possuem em sua estrutura molecular elétrons no estado fundamental (S0) que passam para um estado excitado (S1) após a absorção da energia UV de um fóton (PELISSARI et al., 2003). Para o elétron voltar ao seu estado natural, é necessário que ocorra a liberação da energia absorvida, realizada na forma da liberação de um fóton de menor energia que o absorvido (Figura 1). Ou seja, o alvejante aumenta o comprimento de onda da radiação UV incidente refletindo-a como radiação visível.

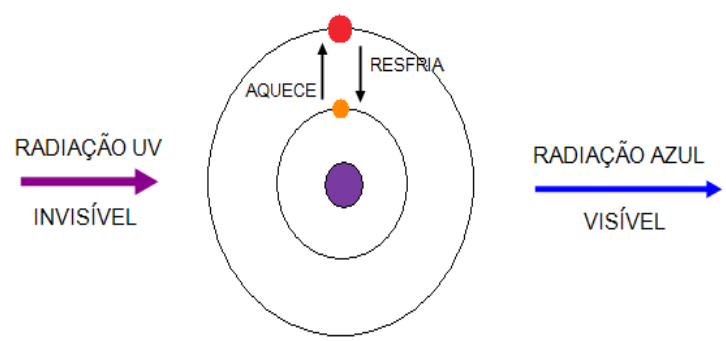

Figura 1 - Fenômeno de fluorescência.

Figure 1 - Fluorescence phenomenon.

Barbosa (2007), comparando o uso de alvejantes dissulfonado e tetrassulfonado em aplicação na massa, relatou que o uso do dissulfonado possibilita uma diminuição de $13 \%$ na dosagem total de alvejantes, o que representaria uma economia de $9 \%$ do custo de alvejantes. Várias fábricas no Brasil obtiveram sucesso na substituição do alvejante tetrassulfonados pelo dissulfonados nesse tipo de aplicação.

Além dos fatores que influenciam a adsorção das moléculas de alvejante, a eficiência do branqueamento ótico depende também das características da celulose. Conforme demonstrado por Eiras et al. (2005), as polpas branqueadas com estágio final de peróxido, por exemplo, apresentam maior estabilidade de alvura, favorecendo a eficiência do alvejante. Quanto à alvura, quanto maior, mais eficiente é o tratamento com alvejante (ZHANG et al., 2007b). Quanto ao tipo de polpa, os alvejantes têm menos afinidade às polpas de alto rendimento do que à polpa química (ZHANG et al., 2007b).

Algumas substâncias podem ainda limitar a ação dos alvejantes ao reagirem com o mesmo ou absorverem a radiação ultravioleta incidente. Holmberg (1999) menciona que diversos alvejantes podem ser oxidados por substâncias oxidantes presentes na polpa. A lignina, por exemplo, tem forte afinidade com radiação UV, o que resulta numa competição com o alvejante (PELISSARI et al., 2003; SHI et al., 2012). O mesmo ocorre com a presença de dióxido de titânio encontrado em pastas de alto rendimento (FERREIRA, 2004; SHI et al., 2012). Segundo He et al. (2009), a lignina também absorve a coloração azulada emitida pelo alvejante reduzindo ainda mais a sua eficiência.

A influência da sequência de branqueamento sob a ação dos alvejantes óticos pode existir em decorrência da presença de alguns compostos químicos residuais do branqueamento, como o dióxido de cloro. Os resíduos de cloro podem oxidar a molécula de alvejante. Substâncias que conferem carga iônica ao meio podem também dificultar a adsorção do alvejante nas fibras celulósicas. Íons metálicos como o $\mathrm{Al}^{3+}$ e o $\mathrm{Fe}^{3+}$ reagem com o alvejado limitando a sua eficiência (HOLMBERG, 1999).

Neste estudo, objetivou-se investigar os efeitos da sequência de branqueamento na eficiência dos tratamentos com alvejantes óticos, determinar as causas dos efeitos observados e indicar qual sequência de branqueamento da celulose possibilita maior eficiência do alvejante ótico.

\section{MATERIAL E MÉTODOS}

\subsection{Branqueamento}

Foi utilizada polpa de eucalipto industrial prébranqueada com oxigênio. A polpa foi branqueada em laboratório adotando-se quatro sequências convencionais de branqueamento, sendo elas D(EP)DD, Dht(EP)DD, Dht(EP)DP e Z/(EP)DP. Em cada sequência foram produzidas polpas com alvura de 88,90 e $92 \%$ ISO.

As etapas com dióxido de cloro (D) e peróxido de hidrogênio $(\mathrm{P})$ foram realizadas em sacos de polietileno com o equivalente à $250 \mathrm{~g}$ de polpa secas. Após a adição dos reagentes e homogeneização manual, a polpa foi aquecida e mantida em banho de vapor com controle de temperatura durante o tempo de reação. A etapa com

Cerne, Lavras, v. 20, n. 2, p. 223-230, abr./jun. 2014 
peróxido de hidrogênio pressurizada (EP) foi realizada em reator Quantum - Mark V (reator de teflon) com amostras equivalentes à $250 \mathrm{~g}$ de polpa seca.

$\mathrm{O}$ branqueamento com ozônio foi realizado em reator rotativo de vidro de $3 \mathrm{~L}$ acoplado a um gerador de ozônio, com amostras equivalentes à $50 \mathrm{~g}$ de polpa seca. A polpa foi acidificada com $\mathrm{H}_{2} \mathrm{SO}_{4} 4 \mathrm{~N}$ até $\mathrm{pH}$ 2,5, desaguada até consistência de $40 \%$ em centrífuga, afofada e depositada no reator. Em seguida, a dosagem preestabelecida de ozônio foi injetada no reator onde a reação foi mantida por 2 minutos.

Para o controle de $\mathrm{pH}$, foram utilizados $\mathrm{H}_{2} \mathrm{SO}_{4}$ ou $\mathrm{NaOH}$. Ao final de cada etapa, foram coletadas amostras do licor para a determinação de carga residual de reagentes. As polpas foram lavadas com o equivalente a $9 \mathrm{~m}^{3}$ de água destilada por tonelada de polpa. Na Tabela 1, apresentam-se as principais condições de cada etapa de branqueamento.

Tabela 1 - Condições do branqueamento.

Table 1 - Bleaching conditions.

\begin{tabular}{ccccc}
\hline Etapa & $\begin{array}{c}\text { Tempo } \\
(\mathrm{min})\end{array}$ & $\begin{array}{c}\text { Temperatura } \\
\left({ }^{\circ} \mathrm{C}\right)\end{array}$ & pH final & $\begin{array}{c}\text { Consistência } \\
(\%)\end{array}$ \\
\hline D0 & 30 & 60 & $2,5-3,5$ & 10 \\
$\mathrm{D}_{\text {hot }}$ & 120 & 95 & $3,0-3,5$ & 10 \\
D1 & 120 & 75 & $3,5-4,5$ & 10 \\
D2 & 120 & 75 & $4,5-5,5$ & 10 \\
$(\mathrm{EP})$ & 120 & 85 & $9,5-10,5$ & 10 \\
$\mathrm{P}$ & 120 & 75 & $9,5-10,5$ & 10 \\
\hline
\end{tabular}

\subsection{Alvejamento}

Foram utilizados dois tipos de alvejantes óticos, sendo um dissulfonado e um tetrassulfonado. As cargas de alvejante utilizadas foram de 2,5 e $8 \mathrm{~kg} / \mathrm{t}$ de polpa seca. Os alvejantes foram diluídos em água destilada, alocados em vidro âmbar e utilizados apenas durante as 8 horas seguintes para evitar perda de eficiência dos alvejantes.

$\mathrm{O}$ alvejante foi adicionado à polpa com consistência de $4 \%$ e mantido sob agitação por 3 minutos. Após a mistura, foram formadas folhas com $85 \mathrm{~g} / \mathrm{m}^{2}$ utilizando água destilada, prensadas e mantidas em sala climatizada $\left(23^{\circ} \mathrm{C}\right.$ e $50 \%$ de umidade) ao abrigo da luz durante o período de secagem.

\subsection{Procedimentos Analíticos}

As leituras de brancura foram realizadas em equipamento Datacolor Spectraflash 500, com observador $10^{\circ}$ e iluminante $\mathrm{D} 65$, de acordo com a norma TAPPI T 560 pm-96 (TECHNICAL ASSOCIATION OF THE PULP AND PAPER INDUSTRY - TAPPI, 1999).

Para a determinação de número kappa e alvura ISO foram adotados os procedimentos da norma TAPPI T 236 om-06 e TAPPI T 452 om-08, respectivamente. As medições de organoclorados da polpa (OX) foram realizadas seguindo a norma Scan W9:89, apenas nas polpas com alvura de 90\% ISO (TAPPI, 2006, 2008).

\subsection{Procedimentos estatístico}

Os procedimentos estatísticos consistiram de modelagem, por meio de análise de regressão realizada com o auxílio do software Curve Expert 4.0. Os modelos gerados são apresentados nos resultados. Inicialmente, foi obtido um modelo para cada sequência de branqueamento, correlacionando a alvura com brancura (na ausência de alvejante). Em seguida, foi gerado um modelo para cada alvejante, em cada sequência de branqueamento, correlacionando a brancura com a carga de alvejante aplicada. Os modelos foram unidos, obtendo-se para cada sequência de branqueamento e tipo de alvejante, um modelo de estimação de brancura em função da alvura inicial da polpa e carga de alvejante ótico.

\section{RESULTADOS E DISCUSSÕES}

Na Tabela 2, apresenta-se a quantidade de reagentes consumidos para o branqueamento das polpas. Para produzir a polpa com alvura de $92 \%$ ISO, a sequência D(EP)DD utilizou de $18,89 \mathrm{~kg}$ de $\mathrm{ClO} 2$ por tonelada de celulose, enquanto a sequência Z/(EP)DP necessitou de apenas $5,7 \mathrm{~kg} / \mathrm{t}$.

Na Figura 2, mostra-se o efeito da dosagem de alvejante dissulfonado na brancura de polpas branqueadas pelas sequências D(EP)DD (figura 2a), Dht(EP)DD (Figura 2b), Dht(EP)DP (figura 2c) e Z/(EP)DP (Figura 2d) em diferentes graus de alvura. Os modelos obtidos para cada sequência estão apresentados na Tabela 3.

O efeito da aplicação de alvejante é bastante acentuado mesmo com pequenas cargas. Com o aumento da carga, a brancura da polpa eleva-se até atingir um ponto de estabilização. Esse fenômeno pode ser explicado pela

Cerne, Lavras, v. 20, n. 2, p. 223-230, abr./jun. 2014 
saturação das fibras. Com o aumento da quantidade de moléculas no sistema, os sítios de ligação fibra-alvejante tendem a se tornarem mais escassos, diminuindo, assim, a adsorção de novas moléculas.

Tabela 2 - Consumo de reagentes no branqueamento.

Table 2 - Consumption of bleaching reagents.

\begin{tabular}{cccccc}
\hline \multirow{2}{*}{$\begin{array}{c}\text { Sequência de } \\
\text { branqueamento }\end{array}$} & $\mathrm{ClO}_{2}$ & $\mathrm{H}_{2} \mathrm{O}_{2}$ & $\mathrm{H}_{2} \mathrm{SO}_{4}$ & $\mathrm{NaOH}$ & $\mathrm{O}_{3}$ \\
\hline D(EP)DD & 12,5 & 3 & 13,4 & 9 & - \\
& 14,3 & 3 & 7,5 & 9 & - \\
& 18,9 & 3 & 0,5 & 10,5 & - \\
$\operatorname{Dht}(\mathrm{EP}) \mathrm{KD}$ & 10,5 & 3 & 11,5 & 9 & - \\
& 12,4 & 3 & 3,75 & 9 & - \\
& 15,5 & 3 & 0,5 & 12 & - \\
$\operatorname{Dht}(\mathrm{EP}) \mathrm{DP}$ & 9,3 & 4 & 7 & 11,5 & - \\
& 10,1 & 5 & 6,7 & 14 & - \\
& 14,3 & 5 & 0 & 14 & - \\
$\mathrm{Z} /(\mathrm{EP}) \mathrm{DP}$ & 1,9 & 10 & 9 & 18 & 5,5 \\
& 3,8 & 10 & 8 & 18 & 5,5 \\
& 5,7 & 10 & 8 & 18,5 & 5,5 \\
\hline
\end{tabular}

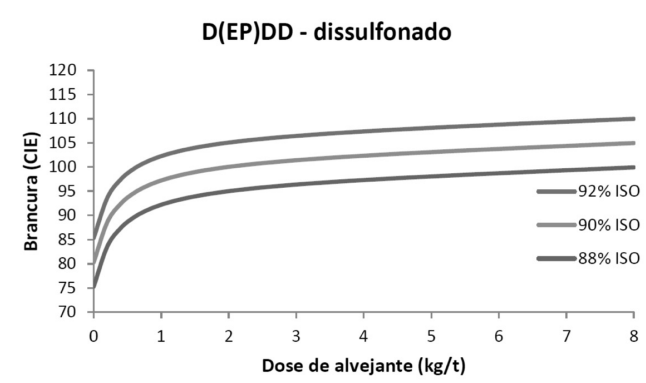

Dhot(EP)DP - dissulfonado

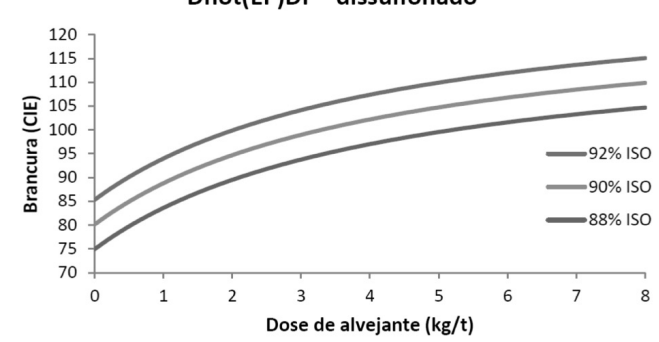

Tabela 3 - Modelos matemáticos para brancura em função de alvura e dosagem de alvejante ótico.

Table 3 - Mathematical models for whiteness as function of brightness and optical brightening agent charge.

\begin{tabular}{cc}
\hline $\begin{array}{c}\text { Sequência de } \\
\text { braqueamento }\end{array}$ & Modelo \\
\hline D(EP)DD & $\mathrm{y}=-145,78+2,51 \cdot \mathrm{X}_{1}+\left(\left(64,92 . \mathrm{X}_{2}\right) /(1+2,89\right.$. \\
& $\left.\left.\mathrm{X}_{2}-0,047 . \mathrm{X}_{2}^{2}\right)\right)$ \\
$\mathrm{D}_{\text {hot }}(\mathrm{EP}) \mathrm{DD}$ & $\mathrm{y}=-127+2,305 \cdot \mathrm{X}_{1}+\left(\left(36,97 . \mathrm{X}_{2}\right) /(1+1,826\right.$. \\
& $\left.\left.\mathrm{X}_{2}-0,025 \cdot \mathrm{X}_{2}^{2}\right)\right)$ \\
$\mathrm{D}_{\text {hot }}(\mathrm{EP}) \mathrm{DP}$ & $\mathrm{y}=-153,28+2,59 \cdot \mathrm{X}_{1}+\left(\left(10,71 \cdot \mathrm{X}_{2}\right) /(1+0,236\right.$. \\
& $\left.\left.\mathrm{X}_{2}-0,0001 \cdot \mathrm{X}_{2}^{2}\right)\right)$ \\
Z/(EP)DP & $\mathrm{y}=-74,8+1,73 \cdot \mathrm{X}_{1}+\left(\left(18,89 \cdot \mathrm{X}_{2}\right) /\left(1+0,52 \cdot \mathrm{X}_{2}-\right.\right.$ \\
& $\left.\left.0,01 \cdot \mathrm{X}_{2}^{2}\right)\right)$ \\
\hline Nota: $\mathrm{X}_{1}:$ Alvura; $\mathrm{X}_{2}:$ Dose de alvejante ótico $(\mathrm{kg} / \mathrm{t})$
\end{tabular}

Nota-se que os comportamento dos modelos das sequências D(EP)DD e Dht(EP)DD são semelhantes entre si, mas diferem do comportamento apresentado pelos modelos das sequências Dht(EP)DP e Z/(EP)DP. As polpas branqueadas pelas sequências $\mathrm{D}(\mathrm{EP}) \mathrm{DD}$ e $\mathrm{Dht}(\mathrm{EP})$ DD apresentaram uma elevação rápida da brancura antes da carga de $2 \mathrm{~kg} / \mathrm{t}$ de alvejante. Com o aumento da carga, a elevação da brancura passou a ser menos pronunciada

Figura 2 - Efeito da sequência de branqueamento, alvura e carga de alvejante dissulfonado na brancura da celulose.

Figure 2 - Effect of bleaching sequence, brightness and disulphonated optical brightener charge on the pulp whiteness.

Cerne, Lavras, v. 20, n. 2, p. 223-230, abr./jun. 2014 
do que a apresentada pelas polpas branqueadas pelas sequências Dht(EP)DP e Z/(EP)DP. A explicação parece estar associada à uma diferença entre esses dois grupos de sequências de branqueamento, o estágio final, que conforme explicado por Eiras et al. (2005), favorece a estabilização da alvura.

O estágio final das sequências D(EP)DD e Dht(EP) DD é realizado com dióxido de cloro, enquanto que nas sequências Dht(EP)DP e Z/(EP)DP utiliza-se o peróxido

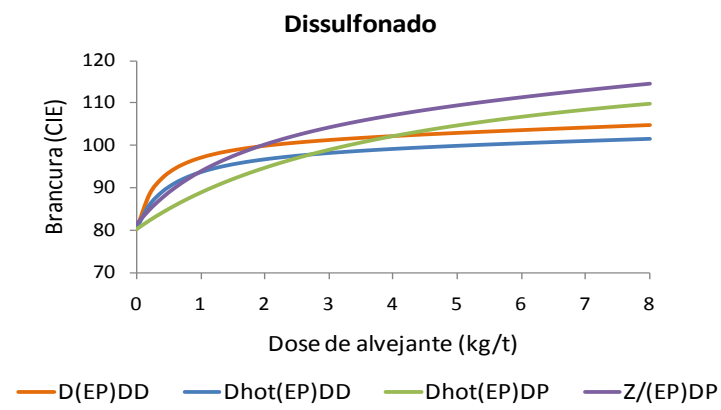

Figura 3 - Influência da sequência de branqueamento da polpa na eficiência de alvejante ótico dissulfonado.

Figure 3 - Influence of the bleaching sequence on the efficiency of the disulphonated optical brightener.
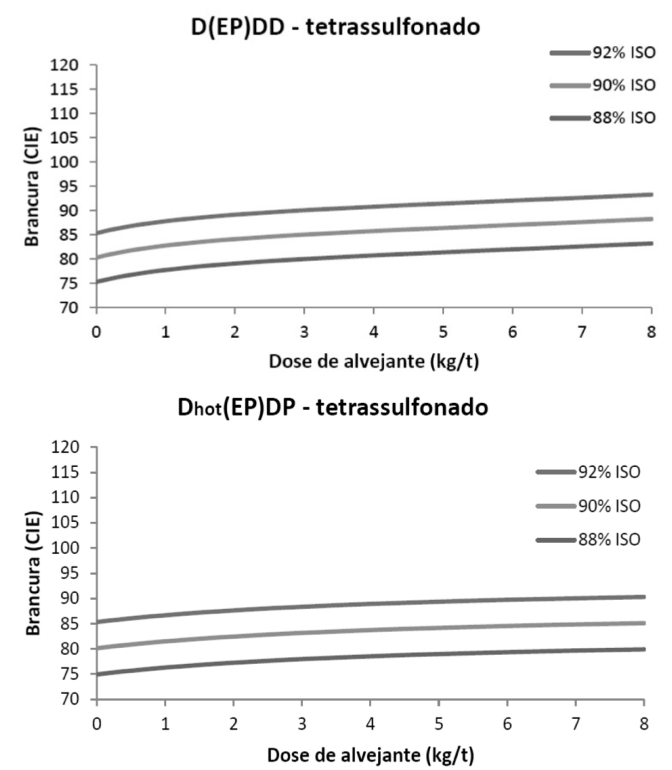

Figura 4 - Efeito da sequência de branqueamento, alvura e carga de alvejante dissulfonado na brancura da celulose.

Figure 4 - Effect of bleaching sequence, brightness and disulphonated optical brightener charge on the pulp whiteness.

Cerne, Lavras, v. 20, n. 2, p. 223-230, abr./jun. 2014

de hidrogênio. Conforme reportado por Holmberg (1999), o cloro é uma substância que pode interferir na ação das moléculas de alvejante ótico. Por ter uma última etapa com dióxido de cloro, polpas branqueadas pela sequência D(EP)DD ou Dht(EP)DD contém traços de cloro residual que interferem na ação dos alvejantes.

Para facilitar a comparação entre as sequências, na Figura 3, comparam-se os efeitos da aplicação de alvejante ótico dissulfonado nas polpas com alvura de 90\% ISO. Os resultados demonstraram que a polpa branqueada pela sequência Z/(EP)DP foi a que apresentou maior brancura após a adição do alvejante, seguida da sequência Dht(EP) DP.

Além da questão do estágio final, existe uma relação inversa entre a carga total de $\mathrm{ClO}_{2}$ utilizada (Tabela 2) e a brancura final (Figura 3). Tal fato evidencia ainda mais a

Tabela 4 - Teor de OX residual das polpas com 90\% ISO.

Table 4 - Level of OX remaining in the pulps at 90\% ISO.

\begin{tabular}{cc}
\hline Amostras & OX $(\mathrm{mg} / \mathrm{kg})$ \\
\hline $\mathrm{D}(\mathrm{EP}) \mathrm{DD}$ & 156,10 \\
$\mathrm{D}_{\text {hot }}(\mathrm{EP}) \mathrm{DD}$ & 130,18 \\
$\mathrm{D}_{\text {hot }}(\mathrm{EP}) \mathrm{DP}$ & 110,98 \\
Z/(EP)DP & 33,19 \\
\hline
\end{tabular}
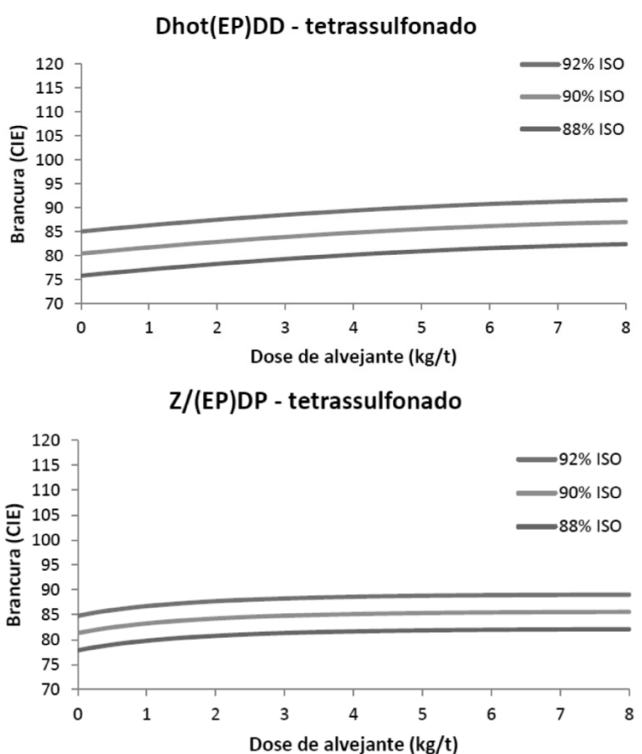
provável interferência do $\mathrm{ClO} 2$ na molécula de alvejante. Para comprovar essa teoria, o teor de organoclorados (OX) nas polpas foi determinado (Tabela 4).

Nas Figuras 4a, 4b, 4c e 4d mostra-se o efeito da dosagem de alvejante do tipo tetrassulfonado na brancura de polpas branqueadas pelas sequências $\mathrm{D}(\mathrm{EP}) \mathrm{DD}$, Dht(EP) DD, Dht(EP)DP e Z/(EP)DP, respectivamente. Em todas as sequências de branqueamento o efeito do alvejante foi relativamente pequeno. $\mathrm{O}$ fato é ligado à baixa retenção dos alvejantes tetrassulfonados na polpa, conforme demonstrado por Barbosa (2007). Embora com maior potencial de fluorescência, alvejantes tetrassulfonado apresentam carga iônica mais negativa do que os alvejantes dissulfonados. Esse caráter eletronegativo reduz a afinidade da molécula com a superfície da fibra, resultando em menor retenção do alvejante. Conforme sugerido por Holmberg (1999), esse fenômeno faz com que a aplicação desse tipo de alvejante idealmente necessite de outros métodos de aplicação, como por exemplo, a aplicação superficial na size-press.

\section{CONCLUSÕES}

As polpas branqueadas pelas sequências com peróxido de hidrogênio no estágio final permitem maior eficiência do alvejante. Sequências de branqueamento que utilizam elevadas cargas de dióxido de cloro e/ou utiliza o dióxido de cloro no estágio final prejudicam a eficiência dos alvejantes óticos.

Dentre as sequências estudadas, a Z/(EP)DP foi a que possibilitou a maior eficiência do tratamento com alvejante ótico.

O alvejante ótico do tipo tetrassulfonado, quando aplicado em massa, apresenta baixa capacidade de retenção, reduzindo o incremento da brancura.

\section{REFERÊNCIAS}

ALÉN, R. Basic chemistry of wood delignification. In: STENIUS, P. (Ed.). Forest products chemistry. Helsink: PI/TAPPI, 2000. p. 59-85.

BARBOSA, C. M. L. Alvejante ótico: avaliação do alvejante di-sulfonade em substituição ao tetrasulfonado em papel de imprimir e escrever. 2007. 38 p. Monografia (Especialização em Tecnologia de Celulose e Papel) - Universidade Federal de Viçosa, Viçosa, 2007.

DENCE, C. W.; REEVE, D. W. Pulp bleaching: principles and practice. Atlanta: TAPPI, 1996. 868 p.

EIRAS, K. M. M.; COLODETTE, J. L.; CARVALHO,
A. M. M. L. Estudo das causas de reversão de alvura de polpa kraft de eucalipto. Cerne, Lavras, v. 11, n. 4, p. 354-368, out./nov. 2005.

FERREIRA, J. C. J. Efeito de agentes branqueadores óticos $x$ reversão de brancura no processo de fabricação de papel couché. 2004. 54 p. Monografia (Especialização em Tecnologia de Celulose e Papel) Universidade Federal de Viçosa, Viçosa, 2004.

FORSSKAHL, I. Brightness reversion. In: STENIUS, P.; PAKARINEN, H. (Ed.). Forest products chemistry. Helsink: PI/TAPPI, 2000. p. 279-324.

HE, Z.; ZHANG, H.; NI, Y.; ZHOU, Y. Adding optical brightening agent to high-yied pulp at the pulp mill. Pulp and Paper Canada, Toronto, v. 110, n. 3, p. 1823, Mar. 2009.

HOLMBERG, M. Dyes and fluorescent whitening agents. In: NEIMO, L. (Ed.). Papermaking chemistry. Helsink: PI/TAPPI, 1999. p. 305-320.

JORDAN, B. Brightness: basic principles and measurement. In: DENCE, C. W.; REEVE, D. W. (Ed.). Pulp bleaching: principles and practice. Atlanta: TAPPI, 1996. p. 695-716.

LEONARDI, L. Brancura e alvura: sua importância para papeis e cartões revestidos. O Papel, São Paulo, v. 51, n. 11, p. 140-141, nov. 1990.

PELISSARI, P. R. B.; SEVRINI, G. I.; HOFFMAN, P. Fatores determinantes no consumo de alvejante ótico na produção de papel branco. In: CONGRESSO INTERNACIONAL DE CELULOSE E PAPEL, 36., 2003, São Paulo. Anais... São Paulo: ABTCP, 2003. 1 CD-ROM.

SHI, H.; LIU, H.; NI, Y.; YUAN, Z.; ZOU, X.; ZHOU, Y. Review: use of optical brightening agent (OBAs) in the production of papers containing high-yield pulps. Bioresources, Oxford, v. 7, n. 2, p. 2582-2591, 2012.

TECHNICAL ASSOCIATION OF THE PULP AND PAPER INDUSTRY. Standard T 236 om-06: kappa number of pulp. Atlanta, 2006.

TECHNICAL ASSOCIATION OF THE PULP AND PAPER INDUSTRY. T 452 om-08: brightness of pulp, paper and paperboard: directional reflectance at $457 \mathrm{~nm}$. Atlanta, 2008.

Cerne, Lavras, v. 20, n. 2, p. 223-230, abr./jun. 2014 
TECHNICAL ASSOCIATION OF THE PULP AND PAPER INDUSTRY. T 560 pm-96: CIE whiteness and tint of paper and paperboard: using $\mathrm{d} / 0^{\circ}$, diffuse illumination and normal viewing. Atlanta, 1999.

ZHANG, H.; HE, Z.; NI, Y.; HU, H.; ZHOU, Y. Effectiveness of optical brightening agent (OBA) on high yield pulps (HYP). In: PAPTAC ANNUAL MEETING, 93., 2007, Monteral. Proceedings... Montreal: PAPTAC, 2007a. 1 CD-ROM.

ZHANG, H.; HU, H.; HE, Z.; NI, Y.; ZHOU, Y. Retention of optical brightening agent (OBA) and their brightening efficiency on HYP-containing paper sheets. Journal of Wood Chemistry and Technology, Philadelphia, v. 27, n. 4, p. 153, 2007 b.

Recebido: 20 de dezembro de 2011; aceito: 23 de agosto de 2013. 
